come alive as people, with all the faults and foibles associated with the human species. The story of the Golden Gate Bridge is principally the story of its chief engineer, Joseph Strauss, and he is both hero and villain of the piece. Strauss was head of an enormously successful Chicago firm that designed and patented bascule bridges of generally modest span and, at best, unremarkable appearance. One such bridge was built in San Francisco around 1916, and it enabled Strauss and the ambitious and influential city engineer, Michael O'Shaughnessy, to cross paths.

O'Shaughnessy had long thought of a bridge across the Golden Gate, and often talked of the idea with engineers whom he met in the course of city business. Before he met Strauss, the estimates O'Shaughnessy received were mostly of the order of a quarter of a billion dollars and full of caveats. Strauss claimed he could build a bridge for under $\$ 25$ million, and in 1921 produced an ungainly design that was priced at $\$ 17$ million. The next lowest estimate was still four or five times as high. Soon Strauss had immersed himself in West Coast politics and cleared the way for financing his project.

How Strauss's ugly duckling evolved into the beautiful Golden Gate Bridge is a fascinating tale. It is complete with revelations about how Charles Ellis, a classics scholar and self-taught bridge engineer, really translated Strauss's conceptual design into an engineering reality. The falling out between Strauss and Ellis, resulting in the latter being denied any official credit for his work on the bridge, was true tragedy.

But the history of the bridge itself, which was designed not so long before the ill-fated Tacoma Narrows Bridge, is a case study of personal and technological adventure bordering on hubris. The relative slenderness of the main suspended span of the Golden Gate was surpassed only by that of the Tacoma Narrows, and in high wind the flexibility of the Golden Gate manifested itself in oscillations not unlike those that were observed in the Tacoma Narrows before it collapsed in 1940. The flexibility of the Golden Gate was also made clear on its recent anniversary by its visible deflection under the weight of a quarter of a million confident and celebrating pedestrians. But flexibility is not failure, of course, and the Golden Gate stands today as a triumph of economical engineering. John van der Zee has captured all of this in a fascinating book that shows that the best of cuttingedge engineering is much, much more than science and technology.

Henry Petroski is a Professor in the Department of Civil Engineering, Duke University, Durham, North Carolina 27706, USA, and author of To Engineer Is Human: The Role of Failure in Successful Design (St Martin's Press, 1985).

\section{Green variations}

\section{Peter D. Moore}

Climate and Plant Distribution. By F. I Woodward. Cambridge University Press: 1987. Pp.174. $H b k £ 22.50, \$ 39.50 ; p b k$ $£ 8.95, \$ 14.95$.

DURING the course of their evolutionary development, botanists have spent an inordinate amount of time and effort on the question of what determines the geographical ranges of plant species and vegetation types. Climate is generally accepted as a major factor in limiting such ranges, but its precise mode of action is often difficult to disentangle from the complex knot of environmental/physiological interactions. Besides the range of physical variables implied by the term 'climate', there are also such matters as scale of study, biological interactions between species and the time dimension to be taken into account if the plant's relationship to climate is to be adequately examined. It is Woodward's aim to deal with this complex subject in the course of a concise book.

Most authors who have tackled vegetation/plant relationships begin with an explanation of the apparently static, frozen moment of the present and then expand into a consideration of vegetation dynamics and climatic change. Woodward chooses the reverse approach and begins his study with the past and the palaeoecological evidence for climatic changes - an interesting but rather dangerous way into the subject. Immediately one is faced with the possibility of circular argument. Fossil records of past vegetation provide evidence of climatic change, and changing climate has resulted in the modification of vegetation. But Woodward carefully circumvents this problem by spending little time on the evidence of fossil floras and vegetation. Instead he concentrates on more direct measures of climatic change, such as the use of oxygen isotope variations in marine sediments and ice cores as an indication of changing ocean and atmospheric temperatures. $\mathrm{He}$ is then in a position to correlate these changes with those observed in the terrestrial fossil record from vegetation and thus avoid circularity in his reasoning. A wise choice of illustrations here has limited consideration to studies where such evidence as pollen data or timberline altitude have been coupled with oxygen isotope data.

Having begun in this way with the time dimension, the consideration of scale clearly has two aspects, spatial and temporal. Of these, the temporal component involves the rate and amplitude of climatic change and the response time of the vegetation. Immediately we are plunged into the problems of population biology, life cycle and, most particularly, generation time of the species in question. Cyclical variation in climate and the incidence of catastrophe are involved in the sifting out of available species in a similar way. Short generation time plants, such as annuals, will be sensitive to short wavelength cycles (of the order of days or weeks), whereas long-lived trees may be sensitive only to cycles of 15 years or more.

The scene is then set to look at global climate, in other words to introduce the spatial dimension, and this is tackled from an energy balance point of view. But even here the time element once more raises its head and climatic change, whether deterministic (such as the Milankovitch cycles) or stochastic (such as changes in the circumpolar vortex), may be expected to play its part in influencing the spatial pattern of vegetation. Translating these climatic variables into vegetation predictions demands one further area of knowledge, namely the ecophysiological responses of the plants themselves, and emphasis is placed upon temperature (influencing life form) and hydrology (influencing leaf area index) as providing a means of predicting vegetation physiognomy on the basis of climatic information. Predictive models, with quite impressive outcomes, are developed on the basis of these two factors. Finally, such additional topics as the latitudinal variation in species diversity, the limitations imposed on taxa by their dispersal mechanisms and the interaction between species in the final struggle for the occupation of a particular location are looked at.

As with other books in the series, this study is not intended to be introductory; it assumes a considerable background knowledge in ecology and physiology. This, coupled with its brevity, means that the author must keep up a hot pace of conceptual development in the text. There is no point at which one can afford to lose concentration; indeed, many sentences need to be read twice in order to absorb their full meaning and implication. But this is in no way a criticism, rather a compliment to the author for his skilful distillation of information.

My main complaint regarding clarity concerns figure captions, which are far too brief to explain the meaning, let alone the significance, of the information the figures contain. Very many of the figures cannot be appreciated without close reference to the text. This is a fault which could easily be remedied in future editions.

Overall, this book must be regarded as an extremely fresh and novel approach to a well worn subject. It is a stimulating, thought provoking and important contribution to the study of climatic controls on plant distribution.

Peter D. Moore is in the Department of Biology, King's College, University of London, Campden Hill Road, London W8 7AH, UK. 